\title{
Implementación de una aplicación para control parental en dispositivos inteligentes
}

Guido Benetazzo Larreátegui / Marco Sotomayor Sánchez

\section{Resumen}

El desarrollo de aplicaciones móviles orientadas al control parental del uso y contenido de dispositivos inteligentes es de gran interés. El presente trabajo fue realizado con el fin de desarrollar un sistema que permita a los padres de familia controlar el uso de los dispositivos inteligentes Android de sus hijos. Para lograr este objetivo primero se efectuó una encuesta para determinar cómo los padres de familia controlan actualmente el uso de los dispositivos de sus hijos y su utilidad. Luego se procedió a desarrollar el sistema y se ejecutaron pruebas del mismo. Finalizado el trabajo se pudo identificar que la mayoría de padres de familia considerarían útil un sistema que les permita realizar esta tarea de manera remota. Una vez puesto a prueba el sistema y en la evaluación comparativa con otros sistemas similares, se pudo concluir que efectivamente la aplicación desarrollada permite gestionar y controlar de manera eficaz, el uso de los dispositivos inteligentes Android

\section{Palabras clave:}

Dispositivos inteligentes, Android, Aplicación, Control parental, Software.

\begin{abstract}
The development of mobile applications oriented to parental control of content and use of intelligent devices is of interest. This paper was written with the objective of developing an application that will help parents manage their children's Android smart devices. The first step to achieve this goal was to make a survey among parents to determine how they currently manage their children's smartphones and if they think that an app is useful. The second step was the actual developing of the application. The third and last step was to test the app in a real case. Once the work was done we found that most parents would consider that an application to manage their children's devices useful. The test in a real case and the benchmark led us to the conclusion that the developed application actually helps managing and controlling the use of Android devices.
\end{abstract}

\section{Keywords:}

Smart devices, Android, Application, Parental Control, Software 


\section{Introducción}

Un estudio realizado por Sánchez-Martínez y Otero (2009) en Madrid a estudiantes de secundaria encontró que el uso intensivo del teléfono móvil y el fracaso escolar están relacionados. Kirshner y Karpinski (2010) de la Universidad de Ohio encontraron que los estudiantes que utilizaban por más tiempo las redes sociales, tendían a tener un promedio menor al de estudiantes que dedicaban menos tiempo a estas actividades. Este dato es relevante, ya que la mayor parte de los accesos a redes sociales se dan a través de dispositivos inteligentes y la tendencia va en ascenso (Chmielewski, 2013) (Dube, 2012).

Dada la evidencia que existe, es necesario crear alternativas para contrarrestar este fenómeno. Actualmente existen varias aplicaciones que se enfocan en el control del contenido que se visualiza en los teléfonos inteligentes como NetNanny (NetNanny, 2012), Kytetime (Kytime, 2015), Norton (onlinefamily, 2015), Kids Place (Google Play, 2015), Screen Time (ScreenTimelabs, 2015) entre otros productos; sin embargo estas herramientas suelen ser complejas de utilizar y de precios elevados, lo cual se convierte en una barrera para que un mayor número de padres de familia se decidan por aplicar este tipo de controles.

El uso intensivo de los dispositivos inteligentes, en especial durante las horas destinadas a los estudios y a la realización de tareas, afecta de manera directa el rendimiento académico de los estudiantes y los hace tres veces más propensos al fracaso escolar (Sánchez-Martínez \& Otero, 2009). Los efectos de este problema son más notables en alumnos que se encuentran cursando la escuela secundaria y la universidad (Stollak, Vanderberg, \& Burklund, 2011). De menor manera también afecta a niños en edades de escuela primaria (González Cortijo, 2012).
En una investigación realizada por el Instituto Nacional de Tecnologías de la Comunicación de España (INTECO, 2011) se encontró que la edad media en la que niños y adolescentes obtienen su primer teléfono móvil se encuentra entre los 10 y 12 años. De acuerdo a la investigación de INTECO, el $82.3 \%$ de menores de edad reportan utilizar sus dispositivos para tomar fotografías, el $77.3 \%$ reporta utilizarlos para escuchar música, El $54.3 \%$ lo utiliza para ingresar a las redes sociales, un $65 \%$ reporta utilizarlos para juegos y el $48.3 \%$ para la mensajería instantánea. Todas estas actividades pueden ser consideradas como distracciones que podrían afectar el rendimiento académico de los menores sin son utilizadas de manera indiscriminada. La investigación también agrega que el control que realizan los padres para el uso de los dispositivos consiste mayoritariamente en normas acordadas verbalmente con los menores, lo cual no garantiza que vaya a ser cumplida.

A nivel local, según estadísticas del Instituto Ecuatoriano de Estadísticas y Censos [INEC] (2013) con respecto al porcentaje de personas entre 5 y 15 años que tienen un teléfono activado en Ecuador, es del $8 \%$, lo cual es un segmento representativo de la población. Teniendo en cuenta los antecedentes, se plantea implementar una aplicación que permita a los padres de familia gestionar el uso de los dispositivos inteligentes de sus hijos de una manera accesible.

\section{Marco teórico}

Definición de teléfono inteligente $y$ Tablet

Joo y Kim (2012) definen a los teléfonos inteligentes como teléfonos móviles de gama alta con capacidades avanzadas de comunicación y procesamiento, los cuales cuentan con unidades centrales de procesamiento (CPU) y unidades de procesamiento gráfico 
(GPU), que son poderosos, con pantallas táctiles de alta resolución y permiten el acceso a información a alta velocidad a través de Wi-Fi, o banda ancha móvil. El diccionario Oxford (2013) define a las tablets como pequeños computadores portables, con los que se interactúa directamente desde la pantalla del dispositivo en lugar de un teclado y un mouse. Se podría decir que las tabletas son computadores muy livianos y portables, conformados por un solo panel cubierto de una pantalla táctil que sirve como el principal medio de interacción, generalmente estas pantallas miden entre 5 y 10.1 pulgadas. La principal diferencia entre teléfonos inteligentes y tabletas radica en el tamaño, siendo las tabletas considerablemente más grandes y debido a esto poseen mayor capacidad de procesamiento $y$ almacenamiento (Cortimiglia, Nogueria, Germán, \& Seben, 2013).

\section{Historia de los teléfonos inteligentes y tablets}

La historia de los teléfonos inteligentes se remonta al año 1993, cuando IBM lanzó al mercado el denominado Simon. Este teléfono contaba con una pantalla táctil y aplicaciones básicas, pero fue un fracaso comercial (Fellet, 2012). En el año 2002 se introdujo la línea de teléfonos BlackBerry, la cual gracias a su enfoque en aplicaciones de correo electrónico se convirtió en un éxito rotundo en el mundo corporativo y posteriormente trasladó ese éxito a todo tipo de consumidores (PCMAG, 2010). Sin embargo no fue hasta el año 2007 que el Iphone de Apple (Apple, 2013) revolucionó el mercado de los teléfonos inteligentes, con su diseño e interfaz intuitiva y lo cambió para siempre (PCMAG, 2010).

A mediados de los años 90 surgieron los primeros experimentos relacionados a computadores en la forma de tableta, pero con malos resultados en varios aspectos claves, especialmente en la parte comercial
(Cortimiglia, Nogueria, Germán, \& Seben, 2013). El concepto de tabletas fue popularizado inicialmente por Microsoft en el año 2001 con el lanzamiento de su denominada "Tablet PC", la cual no obtuvo el éxito esperado. Sin embargo inspiró a varias compañías a lanzar productos similares y a establecer el nombre con el cual se conoce a este tipo de computadores. Al igual que con los teléfonos inteligentes fue Apple quien revolucionó este concepto con el lanzamiento del IPad en el año 2010. Inmediatamente a este acontecimiento varios fabricantes lanzaron sus versiones de tabletas, entre las cuales se destacó la Samsung Galaxy Tab con el sistema operativo Android.

\section{Estadísticas de uso de los teléfonos inteligentes}

En Estados Unidos el $56 \%$ de la población adulta posee un teléfono inteligente (Smith, 2013) (Poulson, 2013). Cada año aumenta significativamente el porcentaje de adultos que poseen este tipo de dispositivos y disminuye el porcentaje de adultos que poseen otro tipo de teléfonos, o ningún tipo de teléfono. Existen diferencias entre los grupos demográficos, en cuanto a la propiedad de un teléfono inteligente, siendo los menores a 54 años los que en mayor proporción poseen este tipo de dispositivos. Esta información es relevante, dado que este grupo demográfico es el que más probabilidades tiene de ser padres de hijos en edades escolares, para los cuales sería útil el software que se propone en este proyecto

En Ecuador aproximadamente 1.2 millones de personas poseen un teléfono inteligente (Ecuadorencifras, 2014). En 2013, el 51,8\% de las personas de 5 años y más poseían un teléfono inteligente. Estas cifras revelan que la penetración de los teléfonos inteligentes en la sociedad ecuatoriana es significativa. 


\section{Sistema operativo Android}

Android es el sistema operativo de mayor crecimiento en el mundo, actualmente en Estados Unidos pasó de tener el $45.4 \%$ del mercado en el año 2012, a tener el 51.2\% en el 2013 (Koetsier, 2013). Android fue creado inicialmente por Andy Rubin, el cual fundó una compañía llamada Android Inc (Lyons, 2010). En el año 2005 Google decidió adquirir esta compañía y mantuvo a Rubin al frente del desarrollo de la misma (Reed, 2010). Para el año 2007 Google anunció oficialmente el lanzamiento de Android, un sistema operativo para dispositivos móviles basado en Linux.

Desde el año de su lanzamiento Android ha ido evolucionando y liberando nuevas versiones del sistema operativo que han mejorado las funcionalidades del mismo. De acuerdo a Ron Amadeo (Amadeo, 2013) del sitio web especializado Android police (s.f.) las versiones son las listadas a continuación:

- 1.0 - Sin nombre

- 1.1 - Petit Four

- 1.5 - Cupcake

- 1.6 - Donut

- 2.0 - Éclair

- 2.1 - Éclair

- 2.2 - Froyo

- 2.3 - Gingerbread

- 3.0 - Honeycomb

- 4.0 - Ice Cream Sandwich

- 4.1 - Jelly Bean

- 4.4 - KitKat

Android está basado en el núcleo de la arquitectura del sistema operativo Linux y está compuesto por varias capas, las cuales proveen todas las herramientas requeridas para su funcionamiento correcto (Google Inc, 2014) . La arquitectura de Android es la siguiente:

\section{Figura 1.}

Arquitectura de Android.

\section{A. Android Architecture}

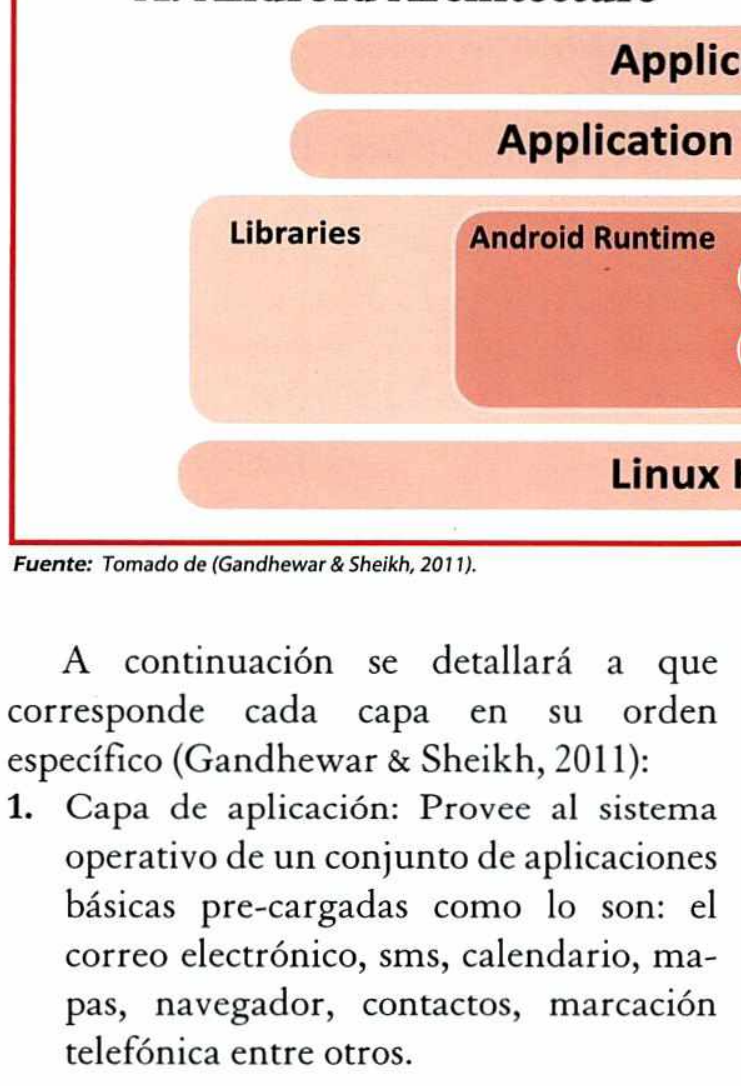

2. Marco de aplicación (Application Framework): Es el marco que permite a los desarrolladores implementar la estructura estándar de una aplicación.

3. Librerías: Librerías escritas en $\mathrm{C} / \mathrm{C}_{++}$ las cuales se invocan a través de una interfaz Java.

4. Android Runtime: la cual está compuesta por un conjunto de librerías que 
proveerán la mayor parte de las funcionalidades disponibles en las librerías java comunes y por la máquina virtual Dalvik, que se encarga de interpretar el código java y ejecutarlo.

5. Núcleo Linux: Finalmente se encuentra el núcleo de Linux el cual permite manejar temas como la seguridad, manejo de memoria, manejo de procesos y otros temas que conciernen al sistema operativo.

Efectos negativos del uso excesivo de los dispositivos inteligentes

El uso de los teléfonos inteligentes en niños y adolescentes va en ascenso; la tasa de penetración de los teléfonos inteligentes en Corea del Sur en niños en edades entre los 6 y 19 años es del 65\%. En Estados Unidos el $37 \%$ de adolecentes ya poseía un dispositivo inteligente para el año 2012 y la tendencia es similar en muchos países (NAM, 2013). El uso indiscriminado y sin control de cualquier aparato, o sustancia puede tener efectos negativos en una persona y esto incluye el uso de dispositivos inteligentes.

El aprendizaje del uso de las Tecnologías de Información y Comunicación (como son los teléfonos inteligentes y las tabletas) y su uso en las aulas de clase es algo necesario en la actualidad. En algunos países como España existe legislación que garantiza la enseñanza de estas tecnologías en la educación primaria; sin embargo es importante fomentar el uso adecuado de los dispositivos ya que de lo contrario estas herramientas podrían afectar en la calidad del tiempo de estudio y disminuir la concentración de los estudiantes (González Cortijo, 2012). En el Ecuador, hasta lo mejor de nuestro conocimiento no hay políticas o estudios profundos acerca del uso adecuado de teléfonos inteligentes en niños $\mathrm{y}$ adolescentes.

A medida que va aumentando el uso de los teléfonos inteligentes también aumentan los problemas por el uso excesivo de los mismos. Los problemas por el abuso de estos dispositivos son variados, los cuales incluyen problemas de aislamiento social, síntomas depresivos, dependencia al teléfono, uso de drogas y alcohol, acceso a contenidos inapropiados, entre otros (NAM, 2013). En muchos casos, adolecentes han reportado sentirse inseguros y ansiosos cuando son obligados a estar sin el dispositivo, o si por alguna razón salen sin él (Merry, Domlija, \& Mackenzie, 2005) (Choliz, Villanueva, \& Choliz, 2009).

En los niños y adolescentes el uso excesivo de los teléfonos inteligentes puede ser especialmente perjudicial para el rendimiento académico. Debido a la gran cantidad de aplicaciones disponibles, estos aparatos se convierten en una fuente importante de distracciones, pudiendo perjudicar el desempeño de los estudiantes, especialmente si es usado durante las horas de clase, o durante las horas destinadas para el estudio. En la investigación realizada por Sánchez-Martínez y Otero (2009) a alumnos de secundaria en la ciudad de Madrid, España, se encontró que el $54.8 \%$ de los estudiantes llevaban su celular al colegio y que el $83 \%$ de los que lo llevaban lo usaba durante las clases. En el mismo estudio se encontró que el $41.7 \%$ utilizaba el teléfono de manera intensiva y que un $20 \%$ sufría de algún tipo de dependencia hacia el teléfono celular. Por último el estudio encontró que de los alumnos que tenían problemas de uso excesivo del teléfono inteligente un $31.8 \%$ había experimentado algún nivel de fracaso escolar (perder un año o reprobar materias). Estos datos son reveladores y muestran lo propenso que son los niños y adolescentes a caer en un mal uso de estos dispositivos, generando consecuencias graves para su desarrollo normal.

\section{Diseño y metodología de la investigación}

El enfoque principal de este trabajo es el desarrollo del software planteado para resolver el problema identificado. Se utilizará 
un diseño de investigación descriptivo para poder identificar como los padres de familia controlan actualmente el uso de los dispositivos inteligentes de sus hijos y si considerarían útil el uso de un software para el control de estos. También para recopilar información de la experiencia de un padre de familia utilizando el software desarrollado para controlar el uso del dispositivo de su hijo/a.

Para conocer como los padres de familia controlan el uso de los dispositivos de sus hijos y si considerarían útil hacer uso de un software para realizar esta tarea, se aplicará la técnica de la encuesta y con los resultados de la encuesta, se podrá identificar si los padres de familia ven como una prioridad poder controlar el uso de los dispositivos de sus hijos y si estarían dispuestos a utilizar un software que los ayude a realizar esta tarea de manera remota. Esta encuesta permitirá evidenciar si los padres de familia consideran importante poder controlar los dispositivos de sus hijos y si efectivamente considerarían hacer uso de un software para esta tarea.

Para conocer si el software cumple el objetivo de poder gestionar el uso de los dispositivos de los hijos se ejecutará una prueba piloto. En este piloto participará un padre de familia con su hijo, a quienes se les instalará la aplicación en sus respectivos dispositivos y procederán a probar la aplicación durante un periodo de dos semanas. Posteriormente se efectuará una entrevista para conocer la experiencia del padre de familia al utilizar el software. Adicionalmente se realizará un estudio comparativo de las funcionalidades del sistema propuesto, con las de sistemas similares actualmente en el mercado.

La encuesta se cumplirá con un muestreo no probabilístico, se seleccionará 150 padres o madres de familia de ciudadelas ubicadas en la ciudad de Guayaquil y sus alrededores. Se obtendrán los correos electrónicos de los padres seleccionados y la encuesta se realizará a través de internet, utilizando las herramientas de google docs. Las preguntas en la encuesta serán de tipo cerrada, ya que lo que se desea es cuantificar si existe la necesidad del software.

El desarrollo del sistema se cumplió, de acuerdo a las mejores prácticas de google para el desarrollo de aplicaciones en Android.

\section{Arquitectura y funcionamiento general del sistema}

El sistema desarrollado para este trabajo de titulación consta de cinco componentes:

- Aplicación para padres

- Aplicación para hijos

- Servidor Web

- Servidor de base de datos

- Servidor de mensajería GCM

\section{Figura 2. \\ Arquitectura del Sistemas}

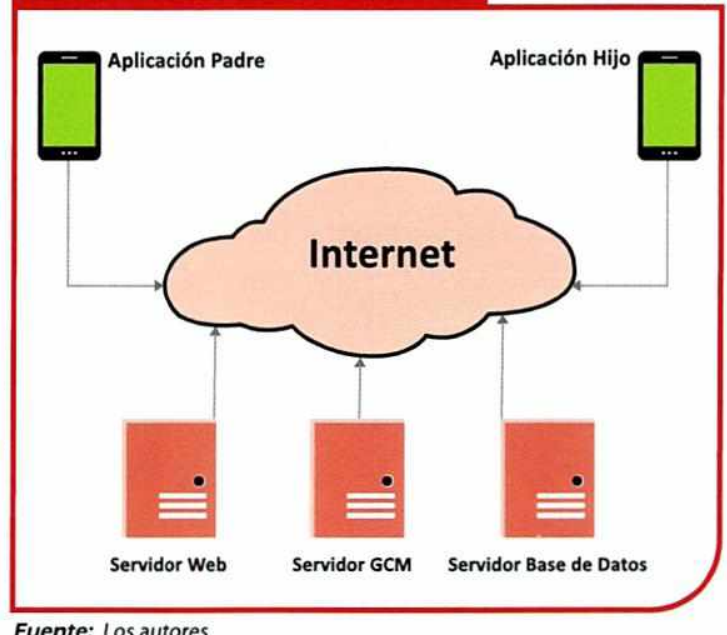

Como se puede ver en la Figura 2, todos los componentes se conectan entre si vía internet. De esta manera se logra que la aplicación funcione en cualquier lugar donde haya acceso a internet. Con esta arquitectura se puede asegurar a un costo inicial bajo, que el sistema tenga alta disponibilidad, siempre y cuando se elijan servicios fiables para alojar los servidores. 
Cada uno de los componentes cumple funciones específicas y tiene su propia estructura. El servidor de base de datos contiene la base principal, la cual almacena la información de todos los usuarios del sistema. La aplicación padre contiene la interfaz de usuario para realizar el control sobre los dispositivos de los hijos. La aplicación hijo contiene una interfaz y varios servicios para seguir las directrices ordenadas por la aplicación padre. Adicional a las dos aplicaciones (Padre e Hijo) cuentan con una base de datos interna que se sincroniza cuando es requerido con el servidor de base de datos principal. De esta manera se optimiza el uso del internet, logrando que no sea requerida una conexión permanente para el funcionamiento del sistema, sino solo cuando se requiera realizar alguna actualización en las reglas dictadas por la aplicación padre, o algún cambio en las aplicaciones disponibles en el dispositivo del hijo. El servidor GCM es el encargado de informar a las aplicaciones Padre e Hijo que se debe realizar una actualización en sus bases de datos internas, esto se logra mediante mensajes enviados vía internet, el cual es recibido e interpretado por cada una de las aplicaciones.

La aplicación guarda toda la información pertinente a su cuenta dentro de una base de datos interna. Esto se realizó con el fin de optimizar el uso de internet y a su vez hacer a la aplicación más rápida de ejecutar, ya que la mayor parte de la información la obtiene localmente. El motor de base de datos que utiliza es SQLite, el cual está optimizado para trabajar en dispositivos con capacidades limitadas y sin necesidad de que sea un servidor aparte, ya que escribe directamente en archivos ordinarios de cualquier disco. Figura 3 muestra el diagrama Entidad-Relación de la aplicación.

\section{Figura 3.}

Diagrama Entidad-Relación de la aplicación padre

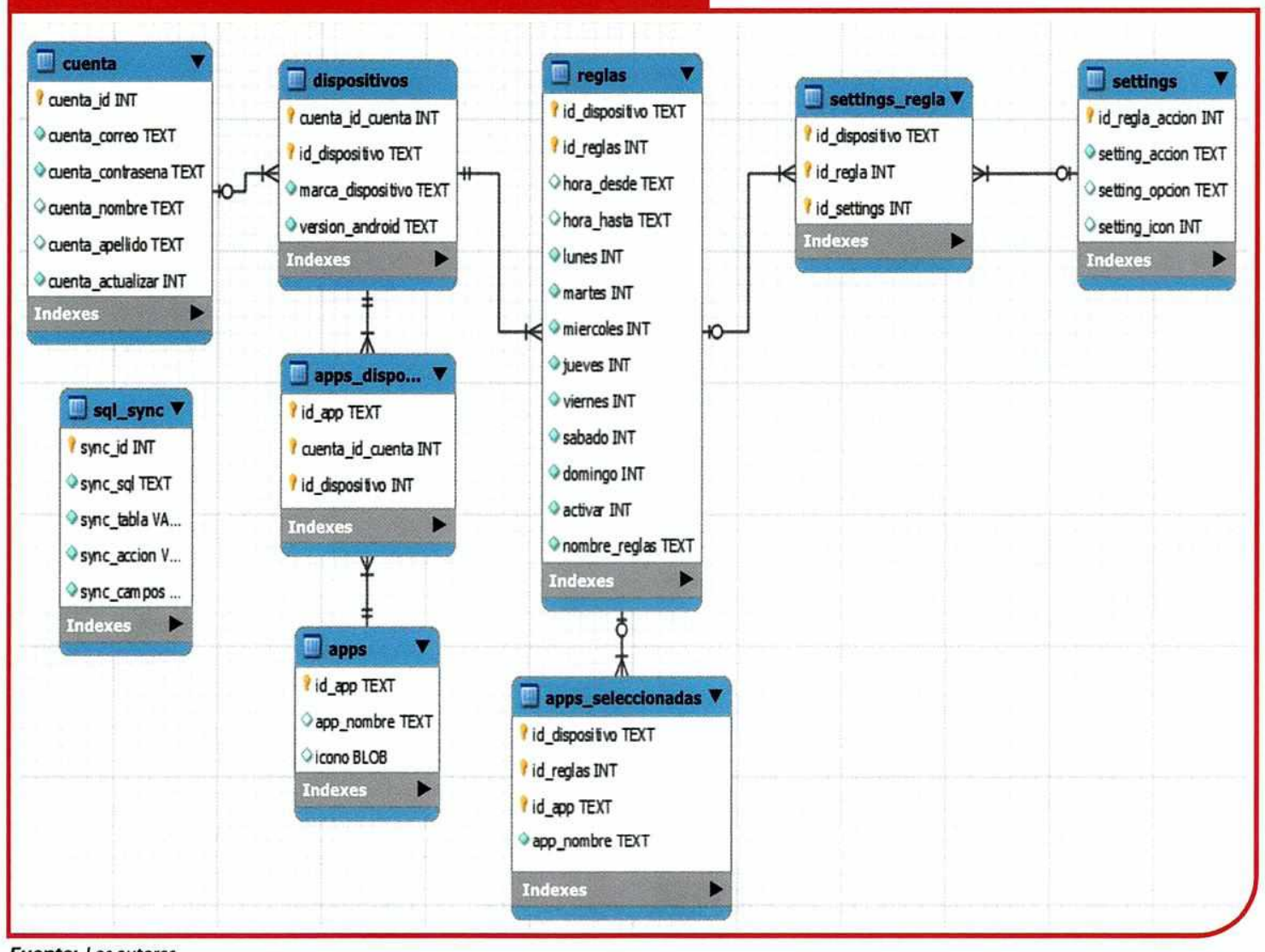

Fuente: Los autores 
Se diseñó el diagrama de clases como se puede apreciar en la Figura 4. Este diagrama de clases contiene las clases más importantes en la aplicación. El detalle de los atributos y de los métodos de cada una de las clases, se lo puede encontrar en la sección de anexos de este documento. En el diagrama se muestra las relaciones y dependencias entre las clases.

\section{Figura 4.}

Diagrama de clases de la aplicación padre

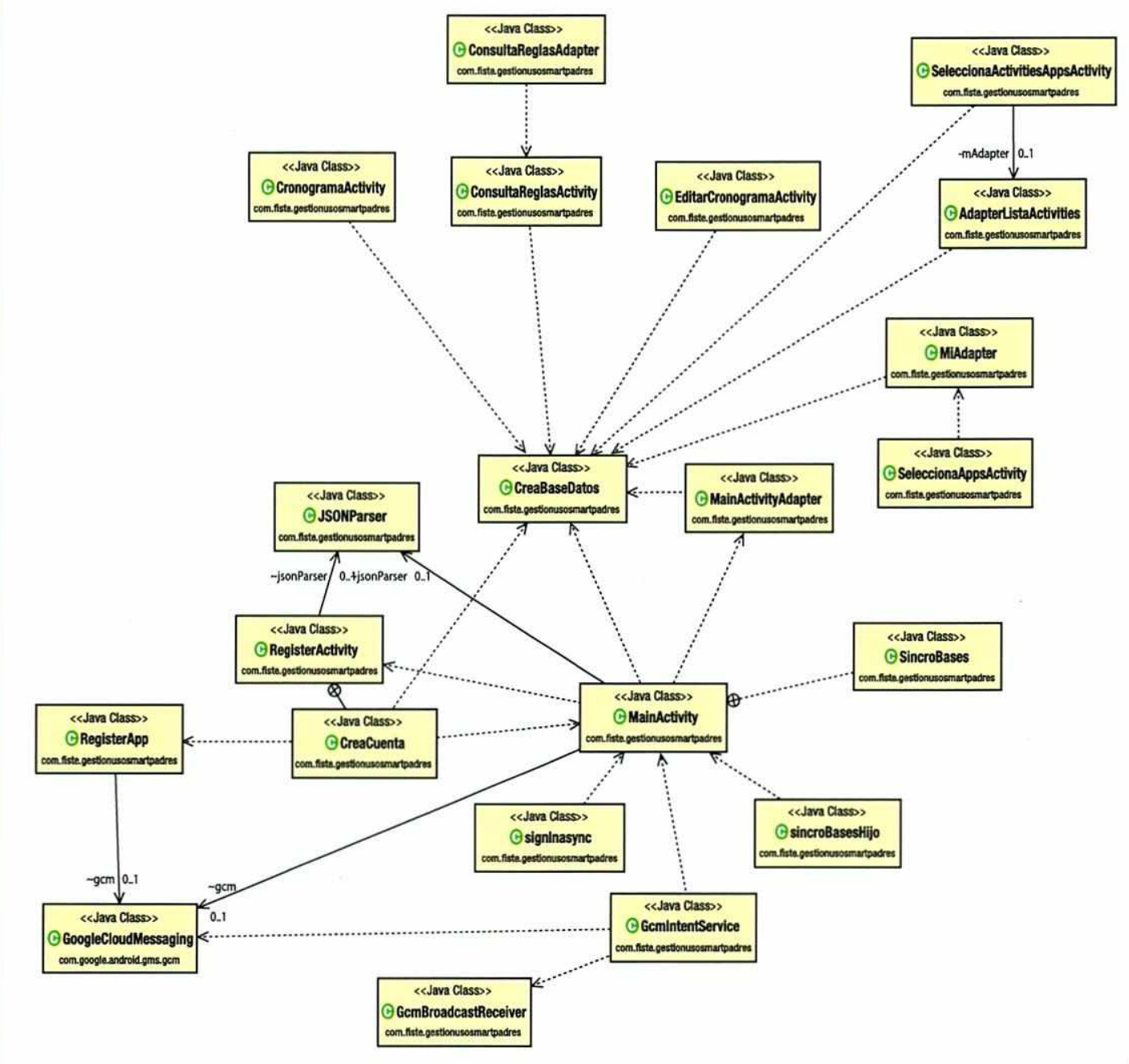

Fuente: Los autores

Después de las fases de diseño, se implementó la aplicación siguiendo las mejores prácticas. En el desarrollo de software es muy importante crear una interfaz de usuario que sea agradable a la vista e intuitiva de utilizar. El diseño de las pantallas se realizó siguiendo las recomendaciones de Google para el desarrollo de aplicaciones en Android. Garantizando que el diseño de las pantallas sigue un estándar al que los usuarios de Android están acostumbrados. De esta manera se puede lograr que la aplicación cumpla con uno de los requerimientos más importantes en las aplicaciones móviles de hoy en día, facilidad en el uso de la aplicación. Figura 5 , muestra algunas pantallas de la barra de acciones de la aplicación. 


\section{Figura 5.}

Barra de acciones en distintas pantallas de la aplicación

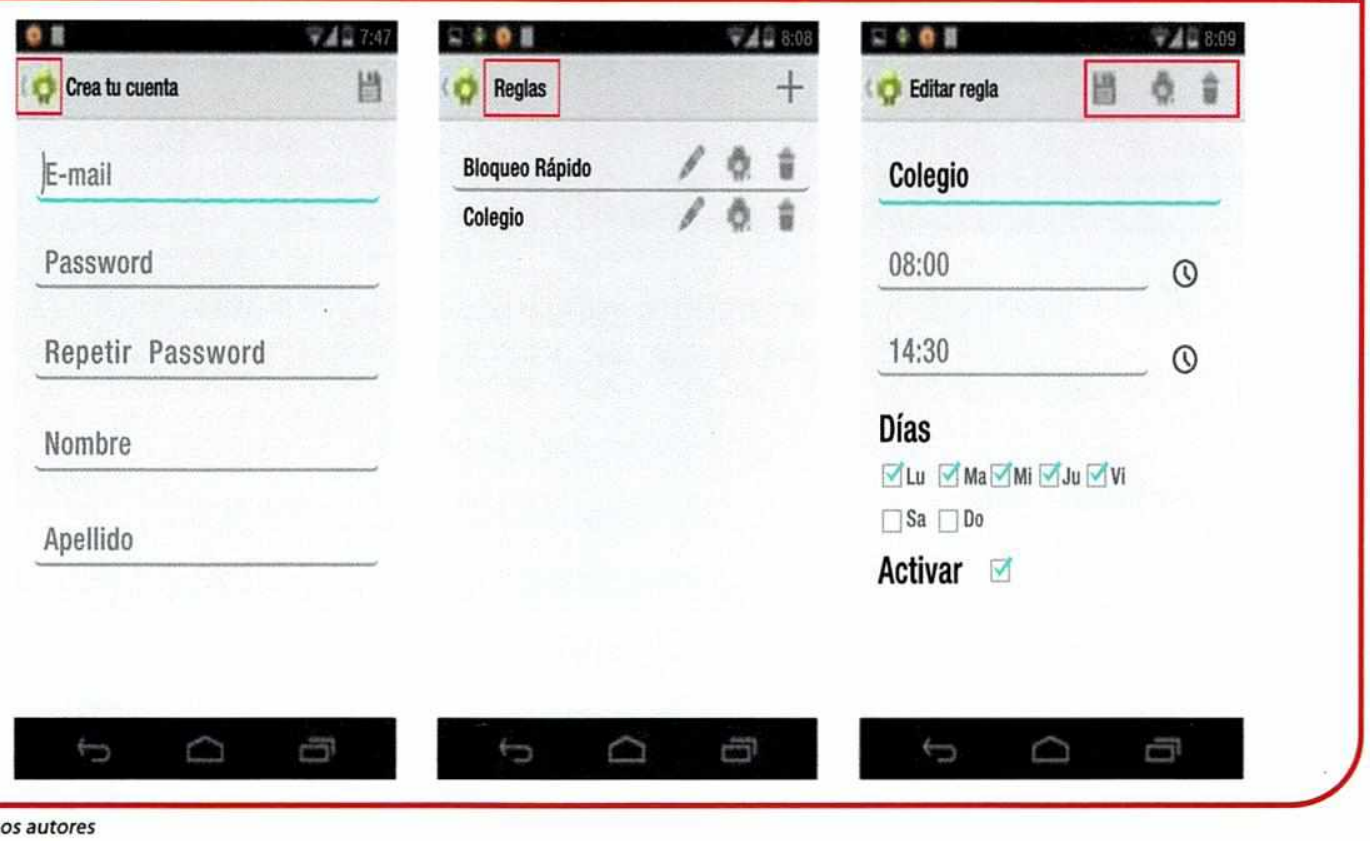

Una vez que la aplicación se realizó, se procedió con la prueba piloto. De los 150 padres de familia seleccionados para la encuesta se seleccionará a un padre de familia para formar parte del piloto, en donde se pondrá a prueba el software por un periodo de dos semanas. Una vez concluido el periodo de prueba del software se programará una cita con el seleccionado para realizar una entrevista acerca de su experiencia al utilizar la aplicación.

Finalmente se realizará un comparativo con dos sistemas de control para dispositivos inteligentes ya disponibles y probados en el mercado similares al sistema propuesto en este trabajo de investigación, con el fin de validar sus funcionalidades y su cumplimiento del objetivo planteado. El comparativo se enfocará en las funcionalidades de cada uno de los sistemas y comprobará si realmente permiten gestionar el uso de distintos tipos de dispositivos inteligentes con el sistema operativo Android.

\section{Resultados}

En base a la encuesta se pudieron obtener los siguientes resultados que serán presentados mediante gráficos.

\section{Figura 6.}

Controla el uso de los dispositivos inteligentes de los hijos

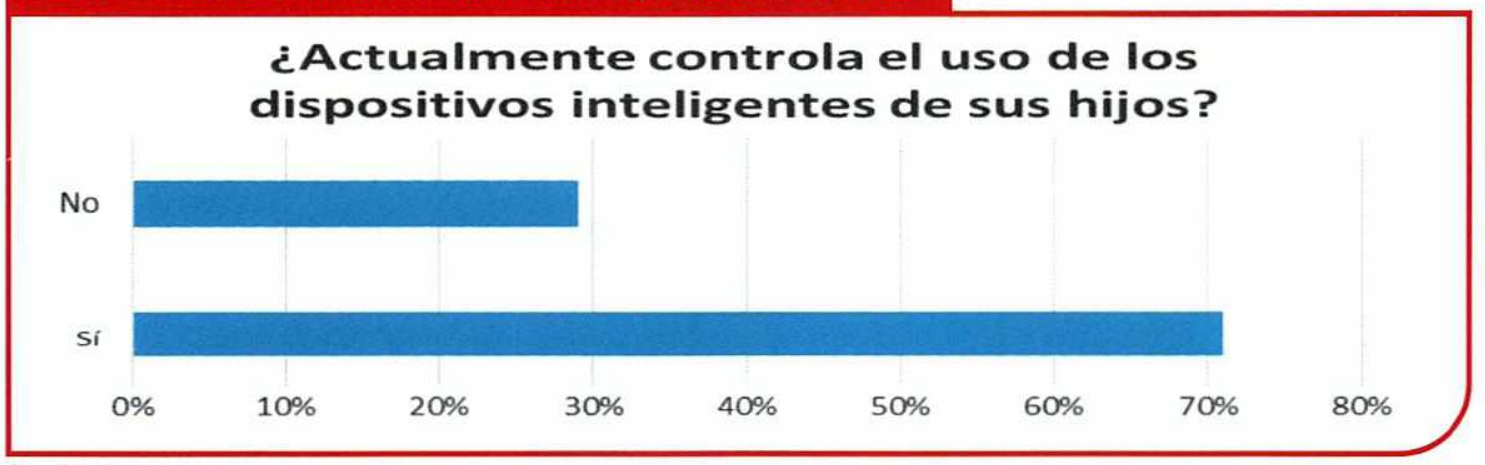

Fuente: Los autores 
En la Figura 6 se puede observar que el $71 \%$ de los padres de familia afirma que controla el uso de los dispositivos inteligen- tes de sus hijos, mientras un $29 \%$ indica que no controla el uso de estos dispositivos.

\section{Figura 7. \\ Formas en las que controlan el uso de los dispositivos inteligentes de los hijos}

\section{¿De qué manera controla el uso de los dispositivos de sus hijos?}

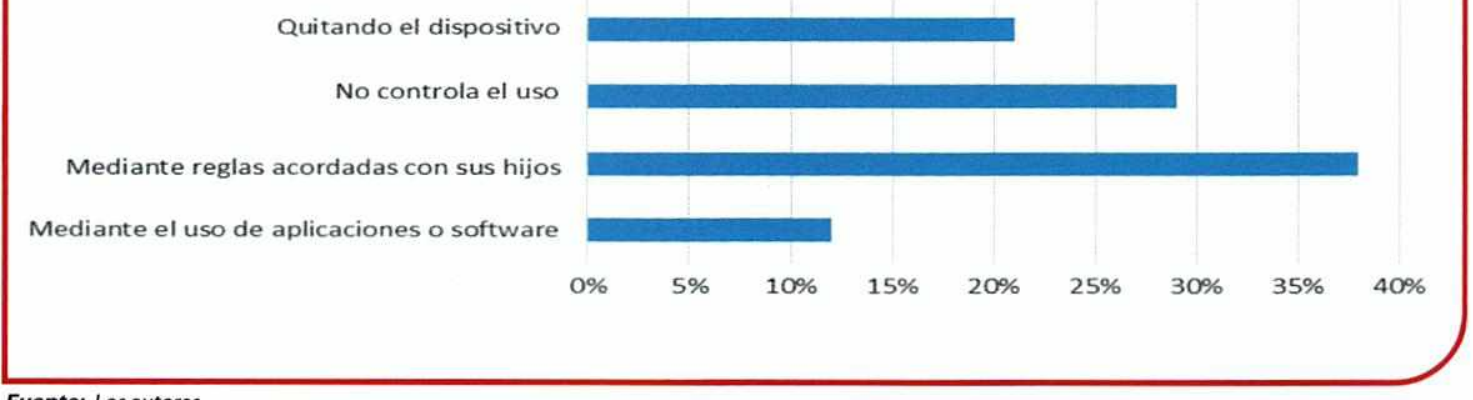

Fuente: Los autores

En la Figura 7 se puede observar los métodos utilizados por los padres de familia para el control de los dispositivos inteligentes de sus hijos. Solo el $12 \%$ de los padres de familia indican que utilizan algún tipo de software para controlar los dispositivos de sus hijos. El $21 \%$ indica que recurre a quitar los dispositivos a sus hijos para poder controlar el uso, esto es poco práctico ya que administrados correctamente son útiles para los padres de familia, especialmente en casos de emergencia para poder establecer comunicación inmediata con sus hijos. Al quitar los teléfonos o tabletas los padres de familia pierden esta funcionalidad importante. El $38 \%$ indica que realiza el control mediante reglas acordadas verbalmente con sus hijos. Es importante establecer reglas, pero es difícil asegurar el cumplimiento de las reglas cuando los hijos no están siendo observados.

\section{Figura 8.}

Los dispositivos inteligentes causan distracciones de las obligaciones de los hijos

\section{¿Su hijo/a se distrae de sus obligaciones debido al uso de algún dispositivo inteligente?}

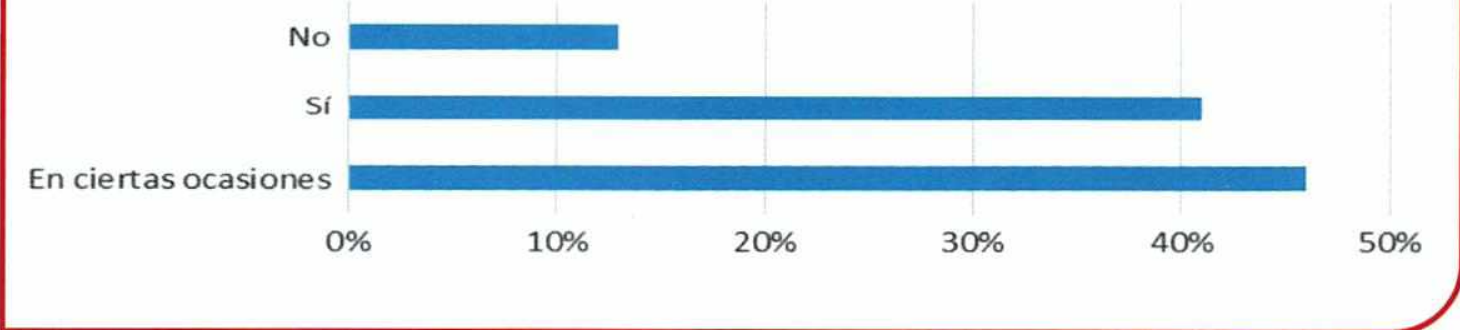

Fuente: Los autores 
En la Figura 8 se puede observar que el $87 \%$ de los padres de familia considera que los dispositivos inteligentes distraen de las obligaciones diarias a sus hijos ya sea siempre, o en ciertas ocasiones.
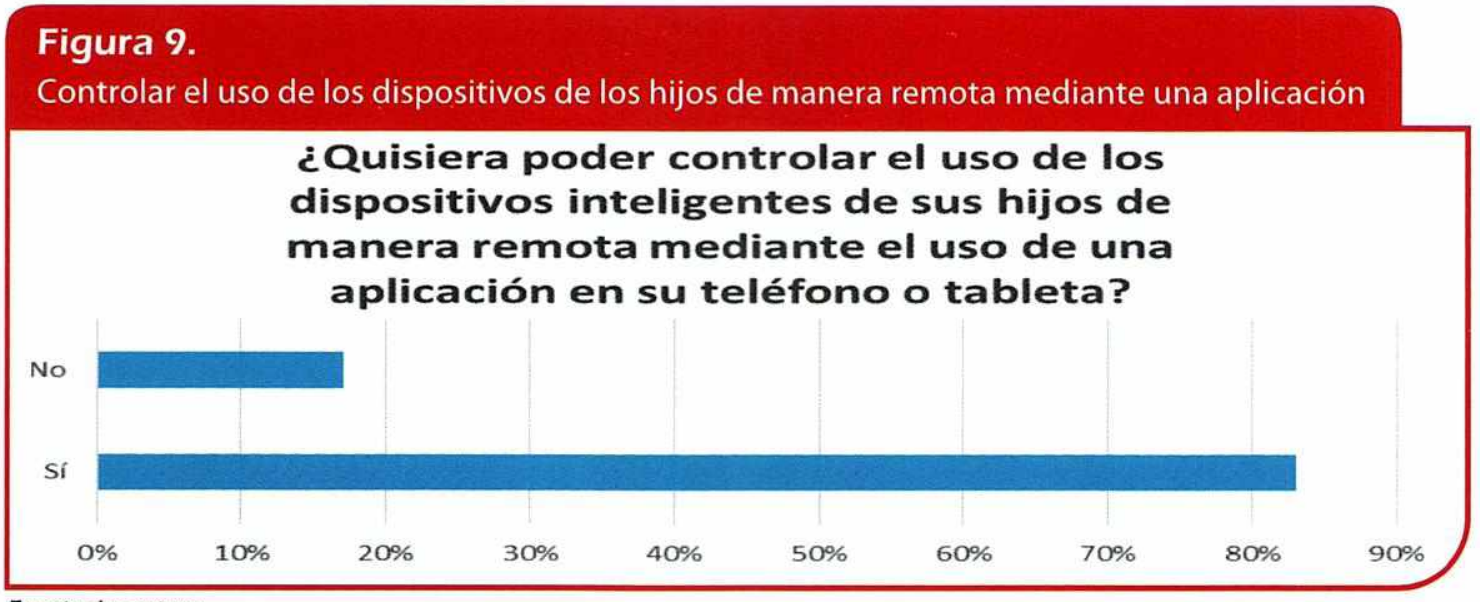

Fuente: Los autores

El $83 \%$ de los padres de familia indican que quisieran poder controlar el uso de los dispositivos de sus hijos de manera remota.

Una vez finalizado el desarrollo de la aplicación se procedió con la prueba piloto con una de las madres de familia encuestadas, con la finalidad de identificar si el sistema cumple el objetivo de eliminar las distracciones causadas por el uso excesivo de los dispositivos inteligentes. El piloto tuvo una duración de dos semanas en la cual la madre de familia pudo hacer uso de todas las opciones del sistema para controlar el dispositivo de su hija. Para la realización del piloto se habilitó el servidor web en el hosting www.000webhost.com, se habilitó el servidor de base de datos y el servidor GCM en los servicios en la nube de google. Se realizó la instalación de la aplicación padre en el dispositivo inteligente de la madre de familia y se realizó la instalación de la aplicación hijo en el dispositivo que iba a ser controlado.

El piloto se ejecutó de acuerdo a lo planificado desde el 5 de enero del 2015, hasta el 18 de enero del 2015. En el periodo de ejecución del piloto se estuvo en contacto diario con el usuario para asegurar que el sistema esté funcionando adecuadamente. Fuera de dudas comunes que pueden surgir cuando se empieza a utilizar un nuevo software, no existieron mayores problemas reportados por parte de los participantes.

Una vez finalizado el piloto se procedió a realizar una entrevista a la madre de familia para conocer su experiencia, utilizando la aplicación y si en su opinión ayudó a disminuir las distracciones de su hija. De acuerdo a lo expresado por la madre en la entrevista el sistema cumplió con el objetivo de permitirle gestionar el uso del dispositivo de su hija y fue útil para eliminarlo como distracción durante las horas de estudio. Adicionalmente la madre de familia expresó que fue de gran ayuda para que su hija evite el uso del dispositivo durante momentos familiares, como las horas de comer o salidas familiares.

Finalmente se realizó una evaluación comparativa con otras aplicaciones disponibles en el mercado, contra la aplicación desarrollada durante este trabajo de investigación. Las aplicaciones escogidas fueron Kids Place y Screen Time, estas dos aplicaciones fueron seleccionadas debido a que sus características son parecidas al sistema que se ha desarrollado y constan entre las más descargadas en la tienda de Android para aplicaciones de esta categoría. La evaluación se enfocó únicamente en las 
funcionalidades básicas de las aplicaciones; es decir aquellas funciones que están destinadas al control de los dispositivos. Las funcionalidades adicionales que pueda tener cada una de las aplicaciones no fueron tomadas en cuenta.

Kids Place es una aplicación de control parental, que se puede convertir en el launcher por defecto del dispositivo inteligente que se desea controlar. El sistema permite bloquear el uso de aplicaciones seleccionadas, definir un tiempo límite de uso al día del dispositivo y mostrar solo las aplicaciones que pueden ser utilizadas. Para evitar que se pueda desinstalar la aplicación, o cambiar las configuraciones Kids Place hace uso de un pin de seguridad que los padres de familia deben definir al momento de instalar el software.

El sistema no tiene una opción de control remoto, por lo que todas las configuraciones deben ser realizadas directamente en el dispositivo que se desea controlar. Screen Time es una aplicación de control parental enfocada en restringir el uso de los dispositivos inteligentes luego de un tiempo límite determinado por los padres de familia. Esta aplicación no reemplaza al launcher por defecto de Android y permite visualizar todas las aplicaciones disponibles en el dispositivo; pero no permite abrirlas si es que el tiempo límite ha sido alcanzado. El sistema permite definir que aplicaciones se bloquearán luego del tiempo límite y también permite definir horarios para dormir y para el colegio, sobre los cuales se puede configurar las aplicaciones permitidas en estos horarios. Para proteger las configuraciones y que la aplicación no sea desinstalada el sistema hace uso de un pin de seguridad. Screen Time permite gestionar el uso de los dispositivos de manera remota ingresando desde una página web.

Como se puede observar en la Tabla 1 las tres aplicaciones tienen funcionalidades básicas similares, pero difieren en ciertos detalles importantes. Los tres sistemas permiten bloquear aplicaciones; de la misma forma permiten definir horarios pero de manera distinta, en este caso solo el sistema propuesto en este trabajo de investigación da la libertad a los padres de familia de establecer horarios personalizados de acuerdo a sus necesidades. Otra diferencia importante es la posibilidad de gestionar muchos dispositivos desde una sola cuenta, lo cual es ideal para padres de familia con más de un hijo, o con hijos que poseen más de un dispositivo inteligente, todo desde una misma consola de administración que puede ser accedida desde cualquier dispositivo Android.

Tabla \# 1

Benchmarking entre aplicaciones de prueba

\begin{tabular}{|c|l|l|l|l|l|}
\hline $\begin{array}{c}\text { Aplicaciones/ } \\
\text { Funcionalidades }\end{array}$ & $\begin{array}{l}\text { Bloquear } \\
\text { Apps }\end{array}$ & Configuración de Horarios & $\begin{array}{c}\text { Control Remotol } \\
\text { Control Local }\end{array}$ & $\begin{array}{c}\text { Mecanismo } \\
\text { de Seguridad }\end{array}$ & $\begin{array}{c}\text { Sestionar } \\
\text { Múltiples } \\
\text { Dispositivos }\end{array}$ \\
\hline Kids Place & $\begin{array}{l}\text { Permite } \\
\text { bloquear } \\
\text { apps }\end{array}$ & $\begin{array}{l}\text { Permite configurar un tiempo } \\
\text { limite de uso al dia }\end{array}$ & $\begin{array}{l}\text { Solo permite control } \\
\text { local }\end{array}$ & $\begin{array}{l}\text { PIN de } \\
\text { seguridad }\end{array}$ & $\begin{array}{l}\text { No permite } \\
\text { gestionar } \\
\text { varios } \\
\text { dispositivos ya } \\
\text { que es de uso } \\
\text { local }\end{array}$ \\
\hline Screen Time & $\begin{array}{l}\text { Permite } \\
\text { bloquear } \\
\text { apps }\end{array}$ & $\begin{array}{l}\text { Permite configurar tiempo limite } \\
\text { de uso al dia y dos horarios } \\
\text { adicionales }\end{array}$ & $\begin{array}{l}\text { Permite control } \\
\text { remoto desde una } \\
\text { página web y control } \\
\text { local }\end{array}$ & $\begin{array}{l}\text { PIN de } \\
\text { seguridad }\end{array}$ & $\begin{array}{l}\text { No permite } \\
\text { gestionar } \\
\text { varios } \\
\text { dispositivos } \\
\text { desde una } \\
\text { misma cuenta }\end{array}$ \\
\hline $\begin{array}{l}\text { Gestión Uso } \\
\text { Smart } \\
\text { (Sistema } \\
\text { propuesto) }\end{array}$ & $\begin{array}{l}\text { Permite } \\
\text { bloquear } \\
\text { apps }\end{array}$ & $\begin{array}{l}\text { Permite configurar horarios } \\
\text { personalizados y a cada } \\
\text { horarios asignar un set de apps } \\
\text { permitidas }\end{array}$ & $\begin{array}{l}\text { Permite control } \\
\text { remoto desde } \\
\text { cualquier dispositivo } \\
\text { Android ingresando } \\
\text { con una cuenta de } \\
\text { usuario y control local }\end{array}$ & $\begin{array}{l}\text { Login con } \\
\text { usuanta de } \\
\text { contraseña }\end{array}$ & $\begin{array}{l}\text { Puede } \\
\text { gestionar } \mathrm{N} \\
\text { dispositivos } \\
\text { desde una } \\
\text { cuenta de } \\
\text { Padre de } \\
\text { familia }\end{array}$ \\
\hline
\end{tabular}


El concepto principal que rige en cada una de las aplicaciones también difiere. Kids Time por defecto bloquea todas las aplicaciones disponibles y solo permite utilizar las aplicaciones que han sido explícitamente permitidas, esto probablemente se debe a que su enfoque primario son los niños pequeños. Screen Time se enfoca en definir un tiempo límite de uso al día y dentro de ese tiempo límite todas las aplicaciones están permitidas. En este caso el objetivo primario de la aplicación es simplemente limitar el tiempo de uso del dispositivo.

El sistema propuesto en este trabajo, por defecto permite utilizar todas las aplicaciones disponibles, pero da la libertad al padre de familia de crear distintos horarios y a cada horario asignarle un set de aplicaciones permitidas, dando así un mayor control al padre de familia, esta diferencia clave en concepto convierte a la aplicación en una opción más versátil que los otros dos sistemas mencionados.

\section{Conclusiones}

Los dispositivos inteligentes son herramientas útiles para todas las personas, incluyendo a niños y adolescentes en edades escolares y colegiales. Sin embargo como ha sido demostrado por varias investigaciones citadas en este documento, también pueden convertirse en distracciones importantes y en ciertos casos en los que existe uso indiscriminado, pueden llegar a convertirse en un gran problema que podría afectar incluso en el rendimiento académico de niños y adolescentes que utilizan este tipo de dispositivos.

La mayoría de padres de familia que poseen dispositivos inteligentes y que tienen hijos que también los poseen, consideran importante controlar de alguna manera el uso de los mismos. De la misma manera consideran útil y necesario tener acceso a algún sistema que les permita controlar el uso de los dispositivos inteligentes de sus hijos de manera remota. Sin embargo, solo un pequeño porcentaje de padres de familia hacen uso de este tipo de sistemas. Este problema en particular fue la base de la idea del desarrollo de una aplicación de control parental para este trabajo de titulación. Una vez finalizado el trabajo se obtuvo el resultado esperado, el cual valida la hipótesis planteada y cumple con el objetivo de este trabajo.

La aplicación efectivamente permite gestionar y controlar el uso de los dispositivos inteligentes Android, lo cual fue comprobado poniendo a prueba el sistema en un caso real con resultados satisfactorios y comparando sus funcionalidades con las de otras aplicaciones similares disponibles en el mercado. Mediante la aplicación de las buenas prácticas de desarrollo de Google se lograron implementar las funcionalidades requeridas y mediante el análisis comparativo con otras aplicaciones similares (Kids Place, Screen Time), se concluye que el sistema implementado en este proyecto tiene ventajas tales como: configuración de horarios personalizados y la gestión de $\mathrm{N}$ dispositivos desde una misma cuenta padre. Es importante resaltar que el software desarrollado puede ser mejorado para ofrecer mayores funcionalidades que podrían ser útiles para los padres de familia. Actualmente el sistema permite a los padres de familia definir aplicaciones permitidas y configurar horarios de uso.

Para convertir el software en un sistema no solo de gestión del uso sino de seguimiento se podrían implementar las si guientes funcionalidades:

*Registrar el tiempo total de uso de cada una de las aplicaciones del dispositivo, *Verificar en tiempo real las aplicaciones que están siendo utilizadas y *Recibir alertas cuando se visualiza contenido no apto para la menores de edad.

El trabajo realizado da una pauta para poder realizar futuras investigaciones sobre el tema. En el plano social se podría realizar un trabajo de investigación que ponga a prueba el sistema aquí planteado y otros 
similares en una muestra significativa de padres de familia e hijos para determinar si efectivamente el uso de este tipo de aplicaciones ayudaría a mejorar el desempeño académico de estudiantes que hayan visto afectado su rendimiento por el uso excesivo de los dispositivos inteligentes y que herramienta o método obtiene un mayor índice de efectividad. En el plano tecnológico se podría realizar un trabajo de investigación que compare el rendimiento y funcionalidad de las distintas herramientas de control parental que existen, para determinar cuál sería el mejor producto en el mercado de este tipo.

\section{Referencias bibliogräficas}

Amadeo, R. (s.f.). Android police. Recuperado el 22 de octubre del 2013, de Android police: http://www.android police.com/2012/09/17/a-history-of-pre-c upcake-android-codenames/

Apple. (2013). Siri. Recuperado el 5 de noviembre del 2013, de Apple: http:// www.apple.com/ios/siri/

Chmielewski, D. (9 de junio del 2013). Los Ángeles Times. Obtenido de Los Ángeles Times: http://articles.latimes. com/2013/ jun/09/entertainment/la-et-ct-nielsen-stu dy-social-networking-smartphone-tablet $-20130609$

Chóliz, M. (2011). MOBILE PHONE ADDICTION: A POINT OF ISSUE. Addiction, 105(2), 373-374.

Cortimiglia, M., Nogueria, F., Germán, A., \& Seben, L. (2013). Tablets: The Next Disruptive Computing Technology? IT Professional, 15(3), 18-25.

Dube, J. (8 de agosto del 2012). Forbes Magazine. Obtenido de Forbes Magazine: http://www.forbes.com/ sites/jondube/ 2012/05/08/why-smartphones-are-the-fu ture-of-social-networking/
Ecuador en Cifras. (2014). 1,2 millones de ecuatorianos tienen un teléfono inteligente (Smartphone). Recuperado de: http://www.ecuadorencifras.gob. ec/12-millones-de-ecuatorianos-tienenun-telefono-inteligente-smartphone/

Fellet, M. (2012). Touch and go. New Scientist, 214(2868), 40-43.

Gandhewar, N., \& Sheikh, R. (2011). Google Android: An Emerging Software Platform For Mobile Devices. International Journal on Computer Science \& Engineering, 12-17.

González Cortijo, I. (2012). Estudio sobre el uso y abuso del teléfono móvil en alumnos de educación primaria. Universidad Internacional de La Rioja.

INTECO. (noviembre del 2011). Instituto Nacional de Tecnologías de la Comunicación. Obtenido de Instituto Nacional de Tecnologías de la Comunicación: www.inteco.es

INEC. (2013). Tecnologías de la información y Comunicación. Recuperado de: http://www.ecuadorencifras. gob.ed documentos/web-inec/Estadisticas_Soci ales/TIC/Resultados_principales_14051 5.Tic.pdf

Joo, B.-G., \& Kim, S.-M. (2012). A User's Experience in Optimizing Smartphone Performance Using Overclocking and Memory Cleaning Techniques. International Journal of Smart Home, 6(4), 127-138.

Koetsier, J. (1 de abril del 2013). Venturebeat. Recuperado el 22 de octubre del 2013, de venturebeat: http://venturebeat.com/ 2013/04/01/android-up-13-ios-down-7-b lackberry-down-81-and-windows-phon e-up-a-massive-52/ 
Kirshner, P., Karpinski, A. (2010). Facebook and academic performance. Computers in Human Behavior

Kytime. (2015). Kytetime Easy Parental Control. Recuperado de http://kytetimeeasy-parental-control.soft112.com/

Lyons, D. (2010). ANDROID INVASION. Newsweek, 156(15), 42-49.

Merry, P., Domlija, D., \& Mackenzie, N. (2005). Global mobile forecasts to 2010. London: Informa Telecoms \& Media.

Google Inc. (8 de octubre del 2014). Android Developers. Obtenido de Android Developers: http://developer.android. com/training/best-ui.html

Google Play. (2015). Kids Place. Recuperado de https://play.google.com/store/apps/ details? id=com.kiddoware.kidsplace\&h l=es_419

NAM, I.-S. (23 de julio del 2013). The wall street journal. Recuperado el 21 de octubre del 2013, de The wall street journal: http://online.wsj.com/news/ articles/SB1000142412788732426340457 8615162292157222

NetNanny. (2012), is your child protected?. Recuperado de https://www.netnanny. com/alt?utm_expid=3852225-27.b_CrIt bERGCRX54D-7Rhgg.1

Onlinefamily. (2015). Norton family. Recuperado de: https://onlinefamily. norton.com/familysafety/loginStart.fs

Oxford Dictionaries. (07 de 10 del 2013). Oxford Dictionaries. Obtenido de http://oxforddictionaries.com/definition /english/smartphone? q=smartphone

Oxford Dictionaries. (07 de octubre del 2013). Oxford Dictionaries. Obtenido de http://oxforddictionaries.com/definition /english/tablet? q=tablet +PC\#tablet_ 9
PCMAG. (08 de octubre del 2010). PCMAG.COM. Obtenido de http:// www.pcmag.com/encyclopedia/term/51 537/smartphone

Poulson, D. (2013). Smartphone Ownership on the Rise. Information Today, 30(7), 10.

Reed, B. (2010). How Android conquered the mobile world. Network World, 27(21), $12-20$.

Sánchez-Martínez, M., \& Otero, A. (2009). Factors Associated with Cell Phone Use in Adolescents in the Community of Madrid (Spain). CyberPsychology \& Behavior, 12(2), 131-137.

Screentimelabs. (2015). Parental control. Recuperado de: https://screentimelabs. $\mathrm{com} /$

Smith, A. (5 de junio del 2013). PewResearchCenter. Obtenido de Pew ResearchCenter: http://pewinternet. org/ Reports/2013/Smartphone-Ownership-2 013.aspx

Stollak, M., Vanderberg, A., \& Burklund, A. (2011). Getting social: The impact of social networking usage on grades among college students. ASBBS, 18(1), 859-865.

\section{Guido Benetazzo Larreátegui}

Ingeniero en Sistemas. Universidad Espiritu Santo - Ecuador.

\section{E-mail: gbenatazzo@uees.edu.ec}

\section{Marco Sotomayor Sánchez}

Ingeniero en Electricidad, especialización Electrónica.

Master of Information Technology - Major in Services.

Docente tiempo completo de la Universidad Espiritu Santo - Ecuador.

E-mail: mvinicio@uees.edu.ec 
